\title{
Slika 2:
}

Bele, Marjan idr. 2014: "A highly active $\mathrm{PtCu}_{3}$ intermetallic core-shell, multilayered Pt-skin, carbon embedded electrocatalyst produced by a scale-up sol-gel synthesis«. Chemical Communications 2014. 50: 13124-13126. 10.1039/C4CC05637J.

https://doi.org/10.1039/C4CC05637J. 31. 1. 2020.

\section{Slika 3:}

Ruiz-Zepeda, Francisco idr. 2019: „Atomically Resolved Anisotropic Electrochemical Shaping of Nano-electrocatalyst «. Nano Letters 2019. 19: 4919-4927. 10.1021/acs.nanolett.9b00918. https://doi.org/10.1021/acs.nanolett.9b00918. 31. 1. 2020. 